\title{
ARC algorithm: a novel approach to forecast and manage daily electrical maximum demand
}

\author{
Da-Chun Wü, Amin Amini ${ }^{a}$, Ali Razban ${ }^{a^{*},}$ Jie Chen ${ }^{a}$ \\ ${ }^{a}$ Indiana University-Purdue University Indianapolis, United States
}

\section{A R T I C LE IN F O}

\section{Article history:}

Received 00 December 00

Received in revised form 00 January 00

Accepted 00 February 00

\section{Keywords:}

Load forecast

Electrical maximum demand

Stochastic model

Peak shaving

\begin{abstract}
A B S T R A C T
This paper proposes an innovative algorithm for predicting short-term electrical maximum demand by using historical demand data. The ability to recognize in peak demand pattern for commercial or industrial customers would propose numerous direct and indirect benefits to the customers and utility providers in terms of demand reduction, cost control, and system stability. Prior works in electrical maximum demand forecasting have been mainly focused on seasonal effects, which is not a feasible approach for industrial manufacturing facilities in short-term load forecasting. The proposed algorithm, denoted as the Adaptive Rate of Change (ARC), determines the logarithmic rate-of-change in load profile prior to a peak by postulating the demand curve as a stochastic, mean-reverting process. The rationale behind this analysis, is that the energy efficient program requires not only demand estimation but also to warn the user of imminent maximum peak occurrence. This paper analyzes demand trend data and incorporates stochastic model and mean reverting half-life to develop an electrical maximum demand forecasting algorithm, which is statistically evaluated by cross-table and F-score for three different manufacturing facilities. The aggregate results show an overall accuracy of 0.91 and a F-score of 0.43 , which indicates that the algorithm is effective predicting peak demand in predicting peak demand.
\end{abstract}

(C) 2014 xxxxxxxx. Hosting by Elsevier B.V. All rights reserved.

\begin{tabular}{|llll}
\hline \multicolumn{2}{|l}{ Nomenclature } & \\
ROC & rate of change & Subscripts and Annotations \\
$\mathrm{X}$ & demand pattern, $\mathrm{kW}$ & $\mathrm{i}$ & index number \\
$\mathrm{q}$ & speed of variation, min $^{-1}$ & $\mathrm{t}$ & time series \\
$\mathrm{n}$ & number of observations & & maximum likelihood estimation \\
$\mathrm{t}$ & time, min & ref & reference \\
$\mathrm{H}$ & mean-reverting half life, minute & & \\
Greek characters & & \\
$\mu$ & mean demand value, $\mathrm{kW}$ & & \\
$\sigma$ & standard deviation & & \\
$\varepsilon$ & aggregated stationary, mean-reverting noise & \\
\hline
\end{tabular}

* Corresponding author. Tel.: +1-317-274-8458; fax: +1-317-274-9744.

Dept. of Mechanical \& Energy Engineering, 723 West Michigan Street, Indinanapolis, IN 46202, E-mail address: arazban@iupui.edu Peer review under responsibility of xxxxx.

This is the author's manuscript of the article published in final edited form as:

Wu, D.-C., Amini, A., Razban, A., \& Chen, J. (2018). ARC algorithm: A novel approach to forecast and manage daily electrical maximum demand. Energy, 154, 383-389. https://doi.org/10.1016/j.energy.2018.04.117 
Table 1 - Summary of the literature survey in electricity demand forecasting and demand-side management

\begin{tabular}{|c|c|c|c|}
\hline Research Characteristics & Methodology Used & Results Obtained & Author Name \\
\hline $\begin{array}{l}\text { Discuss motivations as well } \\
\text { as pros and cons of electricity } \\
\text { demand forecasting } \\
\text { techniques }\end{array}$ & $\begin{array}{l}\text { Present techniques used in } \\
\text { electricity demand forecasting to } \\
\text { increase the efficiency of power } \\
\text { system }\end{array}$ & $\begin{array}{l}\text { Survey and summarize the electricity demand } \\
\text { forecasting techniques and their applications. }\end{array}$ & $\begin{array}{l}\text { Alkhathami et al. [18] } \\
\text { Suganthi et al [19] }\end{array}$ \\
\hline $\begin{array}{l}\text { Load forecasting methods for } \\
\text { power system management }\end{array}$ & $\begin{array}{l}\text { Load forecasting techniques can } \\
\text { be classified as follows: } \\
\text { - Economic approach } \\
\text { - Multiple regression } \\
\text { - Exponential smoothing } \\
\text { - Adaptive load forecasting } \\
\text { - Time series }\end{array}$ & $\begin{array}{l}\text { Main advantage of Econometric approach is that it can } \\
\text { explain why demand can either increase or decrease in } \\
\text { the future. } \\
\text { Main drawback is that electricity changes have to } \\
\text { remain the same. }\end{array}$ & $\begin{array}{l}\text { Feinberg et al. [20] } \\
\text { Hyndman et al. [17] } \\
\text { Kandil et al. [11] } \\
\text { Chikobvu et al. [21] } \\
\text { Amjady et al. [22] } \\
\text { Sigauke et al. [23] } \\
\text { Fan et al. [24] }\end{array}$ \\
\hline $\begin{array}{l}\text { Advanced techniques for load } \\
\text { forecasting }\end{array}$ & $\begin{array}{l}\text { Recent load forecasting } \\
\text { methods: } \\
\text { Neural Networks } \\
\text { End-use Models } \\
\text { Genetic Algorithm } \\
\text { Fuzzy system } \\
\text { Artificial Intelligent Techniques }\end{array}$ & $\begin{array}{l}\text { The most active research in load forecasting has been } \\
\text { neural network. } \\
\text { Neural networks and fuzzy system can model } \\
\text { automatically complex nonlinear input-output } \\
\text { relationships through learning process using a database } \\
\text { of load and explanatory variables. } \\
\text { Artificial intelligent has proven itself as one of the } \\
\text { most reliable techniques. }\end{array}$ & $\begin{array}{l}\text { Islam [15] } \\
\text { Kalogirou [25] } \\
\text { Hippert et al. [26] } \\
\text { Li et al. [27] } \\
\text { Feinberg et al. [20] } \\
\text { Abdel-Aal [28] } \\
\text { Amin-Naseri et al.[29] }\end{array}$ \\
\hline $\begin{array}{l}\text { Demand-side } \\
\text { management/Demand } \\
\text { Response techniques }\end{array}$ & $\begin{array}{l}\text { Particle swarm optimization } \\
\text { (PSO) }\end{array}$ & $\begin{array}{l}\text { HAVC system and manufacturing system are } \\
\text { dependent systems by considering the temperature as a } \\
\text { function of manufacturing operation to find an optimal } \\
\text { demand response strategy. }\end{array}$ & Sun et al. [31] \\
\hline
\end{tabular}

\section{Introduction}

Electrical demand is estimated to increase globally by $85 \%$ in 2040 as economies expand, living standards increase, modernization and industrial development accelerate globally [1]. Electrical demand forecasting plays a pivotal role in power system management, especially for ensuring economic and reliable operation in power systems. To achieve this end, electric utility consumption load forecasting models are needed, to ensure maximizing the load factor to occur at any time, which help the supplied electric energy meet the load requirement plus reducing the energy lost in the power system [2]. Adjusting the supply-demand balance in the electric system at any time requires utilizing different models for load forecasting on a variety of time horizons. Moreover, demand forecasting can be a very strong tool on the costumer side. Load forecasting is categorized based on the time scale and these could be listed into: Very Short-Term Load Forecasting (VSTLF),

Short-Term Load Forecasting (STLF), Medium- Term Load Forecasting (MTLF) and Long-Term Load Forecasting (LTLF). VSTLF is mostly used for load-frequency control and detecting contingencies in power system [2]-[4]. STLF is utilized from an hour-ahead to a day-ahead forecasting in power system operation. MTLF [6]-[9] and LTLF [9]-[11] are ranged respectively from one week to one year, and one year to decades. From the demand side's point of view, demand monitoring and forecasting have become a crucial part of power system in recent years. Demand charge has been increasing dramatically in most dense populated cities. Furthermore, future power systems and smart grids require advanced metering systems to improve the reliability of demand in the conventional power systems.

Many demand forecasting procedures are based on curve-fitting or extrapolation of variables such as weather or economic data. Some utilize more complex algorithm like neural networks or artificial intelligent to produce a model that tries to resemble the original trend curves. Stochastic technique, on the other hand, focuses on only the data itself without considering external variables and is proven effective in random-walk mean-reverting processes [12]-[14]. Although the mathematical and conceptual complexity may deter an energy planner from using stochastic methods, this technique brings a pure form of demand pattern concerning only the mathematical significances. Furthermore, since the stochastic models do not require external data, VSTLF and STLF become possible as data other than electrical load may not be available within a short period of time for the use of other methods.

Utility companies have extensively studied electrical demand forecasting [15]-[17]. Most of the studies proposed the concept and necessity of demand forecasting, techniques for load forecasting, pros and cons, verification of the methods, and their application in the real world. A survey of these researches is presented in Table 1. However, maximum demand forecasting is usually the result of load forecasting in these literatures. Most of the researches were done in two steps, 1) finding the load pattern, and 2) predicting the occurrence of maximum demand. These strategies require more data as input, and intense calculation. In comparison, forecasting the maximum demand on the costumer side, known as "behind the meter", can be as hard as forecasting on utility side because costumer side has much lower load in comparison to utility load. Our literature search resulted in few studies on consumer side forecasting likely due to its randomness and inconsistency. An energy manager requires not only an absolute value of peak but also the ability to know the time when the peak will happen in daily operation. In order to fill these gaps, we propose a new method to provide warning signal prior to an occurrence of a peak demand. 
The object of this paper is to utilize a stochastic technique for producing STLF of daily peak power demands of an electric system. It explores a well-established stochastic approach in forecasting based on the data from previous works in order to warn before a peak occurs. We improve these previous methods by forecasting the time when peaks happen. The ARC algorithm, therefore, is suitable to be integrated in demand response system that sends out warning to the energy management planner.

\section{Proposed Methodology}

A time-series is a sequence of data points, usually consisting of consecutive energy consumption measurements occurred over a time interval [2]. Representing the demand pattern as a time series is generally accepted [31]. Electrical load can be modelled as a class of generalized Ornstein-Uhlenbeck processes and therefore it is a mean reverting process [32]. Together with our short-term assumption listed above we can rewrite a Stochastic Differential Equation (SDE) as given in equation (1).

$$
d X_{t}=q_{t}\left(\mu-X_{t}\right) d t+\sigma_{t} d \varepsilon_{t}
$$

Where $\mathrm{q}_{\mathrm{t}}$ is the speed of reversion; $\mu$ is the mean value; $\sigma_{\mathrm{t}}$ is the standard deviation; and $\varepsilon_{\mathrm{t}}$ represents standard Brownian motion. Given a set of previous observations $\left(\mathrm{X}_{0}, \mathrm{X}_{1}, \ldots, \mathrm{X}_{\mathrm{n}}\right)$, we solve this peak forming equation by providing the maximum likelihood estimators (MLE) as shown in equation (2) [33].

$\hat{q}=-\delta^{-1} \log \left(\frac{n^{-1} \sum_{i=1}^{n} X_{i} X_{i-1}-n^{-2} \sum_{i=1}^{n} X_{i} \sum_{i=1}^{n} X_{i-1}}{n^{-1} \sum_{i=1}^{n} X_{i-1}^{2}-n^{-2}\left(\sum_{i=1}^{n} X_{i=1}\right)^{2}}\right)$

Locally, as far as the deterministic part of the above SDE is concerned we get the ordinary differential equation (ODE), equation (3) by assuming that the differential of $\varepsilon_{t}$ is small when the time interval is small.

$d X_{t}=q_{t}\left(\mu-X_{t}\right) d t$

Integrating equation (3) from $\mathrm{X}_{0}\left(\mathrm{t}_{0}\right)$ to the expected value at the instant $t_{1}$, denoted by $\mathrm{X}_{1}\left(\mathrm{t}_{1}\right)$, and letting $\Delta \mathrm{t}=\mathrm{t}_{1}-\mathrm{t}_{0}$, then we get equation (4).

$\int_{X_{0}}^{X_{1}} \frac{d X_{t}}{\left(\mu-X_{t}\right)}=\int_{t_{0}}^{t_{1}} q_{t} d t$

Equation (4) can be obviously solved by noting the fact that $\mu$ is a constant as given in equation (5)

$q_{t}=-\frac{1}{\Delta t} \log \left(\frac{X_{1}-\mu}{X_{0}-\mu}\right)$

We define logarithmic rate-of-change as the opposite of the local mean reverting rate as shown in equation (6).

$$
R O C_{t} \equiv-q_{t}
$$

And the historical mean reversion rate as the reference is defined in equation (7).

$$
R O C_{r e f} \equiv-\hat{q}
$$

By comparing the current ROC and the reference ROC we can estimate the time of a demand peak as shown in equation (8).

If $R O C_{t} \geq R O C_{r e f}$

Then the peak can happen within the mean reverting half-life, $\mathrm{H}$ as shown in equation (9).

$H=\frac{\log (2)}{q}$

\section{Numerical Simulation}

To further test the robustness and effectiveness of the proposed methodology we constructed a simulated stochastic load process based on an actual demand curve obtained from a manufactory. We used a typical 15-minute average monthly curve as a baseline, which is depicted in Fig. 1.

Fig 1. Mean-demand curve for a weekday in November of a

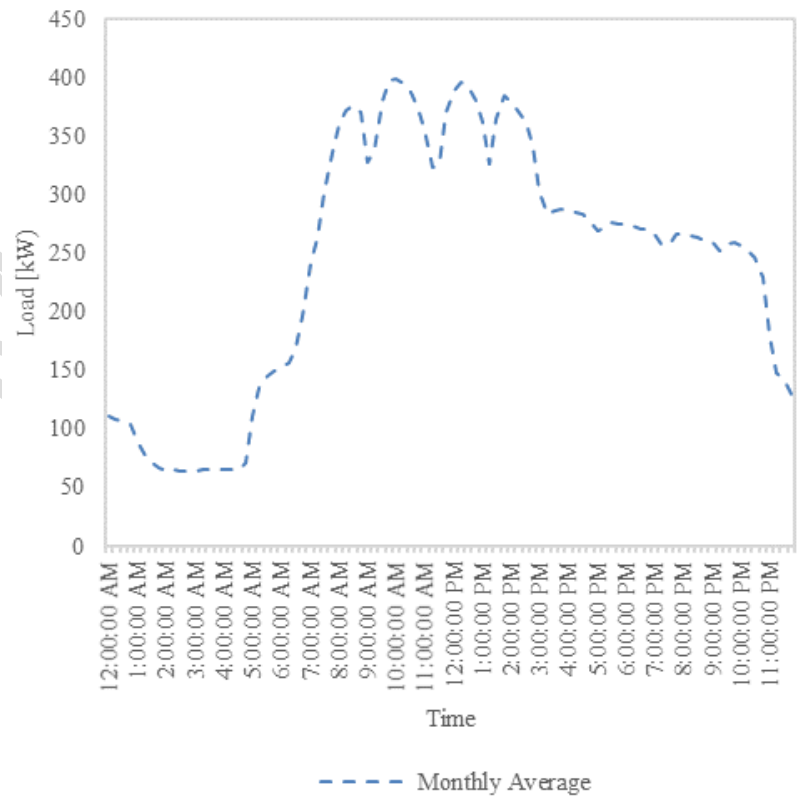

manufactory

To find the mean curve of demand for weekdays (Monday to Friday) in November, the measurement of the demand at the beginning of each weekday was taken and the mean of all measurements was used to calculate the value for the mean-demand at the beginning of that day. Using the mean curve, we simulated a random demand process shown in Fig. 2. The methodology tried to forecast the peak of the simulation and uses the monthly average curve as the historical data. The historical mean curve gives a reference ROC of 0.025 . 


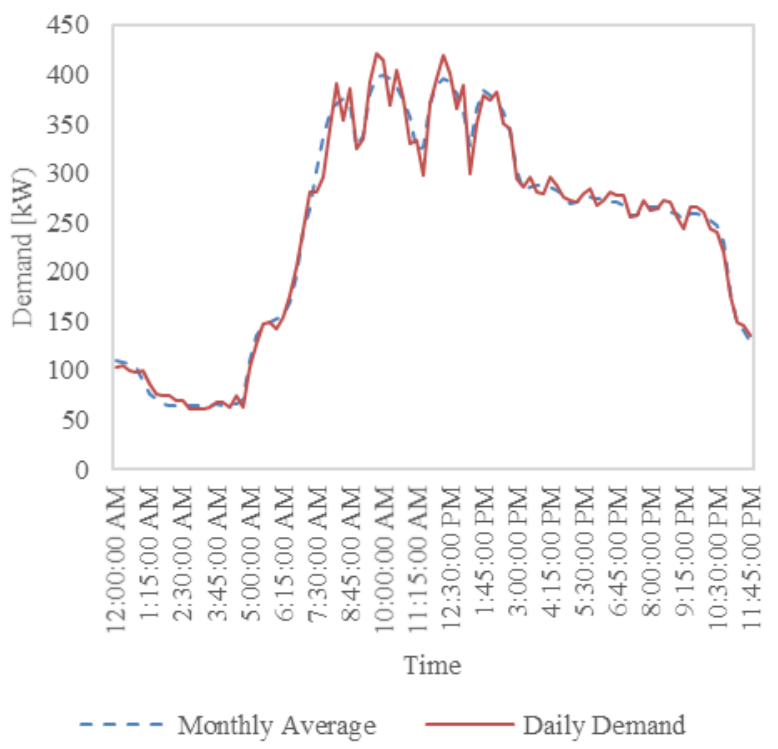

Fig 2. Stochastic simulation of a daily demand.

Using equation (8) and the definition of a peak that it always occurs above the mean, we normalized the numerical simulation against the daily mean value and performed the methodology. A graphical representation of the results is shown in Fig. 3.

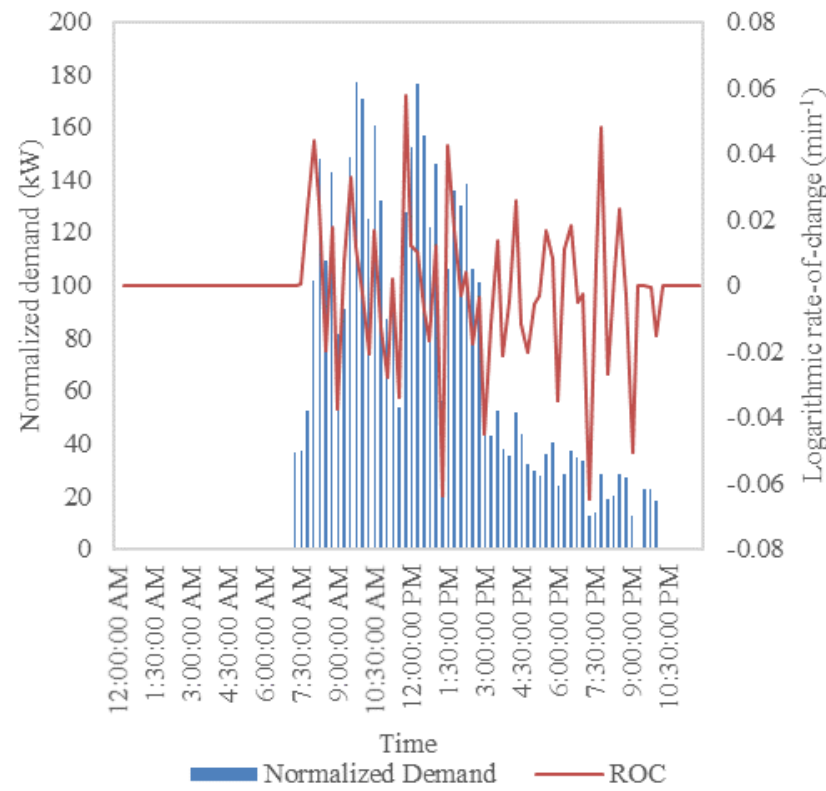

Fig 3. Methodology result from the simulation

Detailed result is tabulated in Table 2. Table 2 shows that by having a mean reverting half-life time of 27 minutes the methodology captured the demand peak at 9:45 PM by signalling a warning at 9:30 PM. However, the result also shows 5 false positive signals.
Table 2. Forecast results from the simulation $\left(\mathrm{ROC}_{\mathrm{ref}}=\mathbf{0 . 0 2 5}\right.$; demand peak = 9:45:00 PM; half-life, $\mathrm{H}=21$ minutes)

\begin{tabular}{llll}
\hline Signal & ROC $\left(\mathbf{m i n}^{-1}\right)$ & Demand $(\mathbf{k W})$ & Result \\
\hline 8:00:00 AM & 0.0440 & 345.51 & False Positive \\
\hline 9:30:00 AM & 0.0329 & 392.37 & True Positive \\
\hline 11:45:00 AM & 0.0576 & 371.07 & False Positive \\
\hline 1:30:00 PM & 0.0424 & 349.59 & False Positive \\
\hline 4:15:00 PM & 0.0256 & 295.61 & False Positive \\
\hline 7:45:00 PM & 0.0481 & 272.08 & False Positive \\
\hline
\end{tabular}

We expanded this test to every month of a calendar year using the same method. In total we performed 12 tests and the model successfully forecasted 10 peaks and missed 2 as shown in Table 3. A sensitivity of 0.83 and a specificity of 0.90 showed the model had good true positive and true negative rates. A precision value of 0.12 means the model creates some false positives. Conventionally, an accuracy of 0.9 represents a good model, but F-score of 0.21 shows the model gives many false positives. We solved the problem by adjusting the threshold.

Table 3. Cross-table of the simulated forecast results $(n=717$; threshold = daily mean)

\begin{tabular}{lll}
\hline \multirow{2}{*}{ Maximum demand forecast } & \multicolumn{2}{l}{ Maximum demand observed } \\
\cline { 2 - 3 } & Yes & No \\
\hline Yes & 10 & 72 \\
No & 2 & 633 \\
\hline Sensitivity & 0.83 & \\
\hline Specificity & 0.90 & \\
\hline Precision & 0.12 & \\
\hline Accuracy & 0.90 & \\
\hline F-Score & 0.21 & \\
\hline
\end{tabular}

We tried to lower the false positive number by performing a sensitivity test regarding to the threshold, below which we do not apply the methodology to the data. Fig. 4 shows a receiver operating characteristic space with different thresholds we have run. Reference [34] suggests choosing model variations that are closest to the upper-right corner. Thus, having a threshold of mean plus 0.5 to 1 standard deviation the model can eliminate some false positives while maintaining a good true positive rate. After adjusting the threshold we improved the precision from 0.12 to 0.22 , while retaining the sensitivity. The F-score was improved by $66 \%$ from 0.21 to 0.35 . An improved result is shown in Table 4 . 


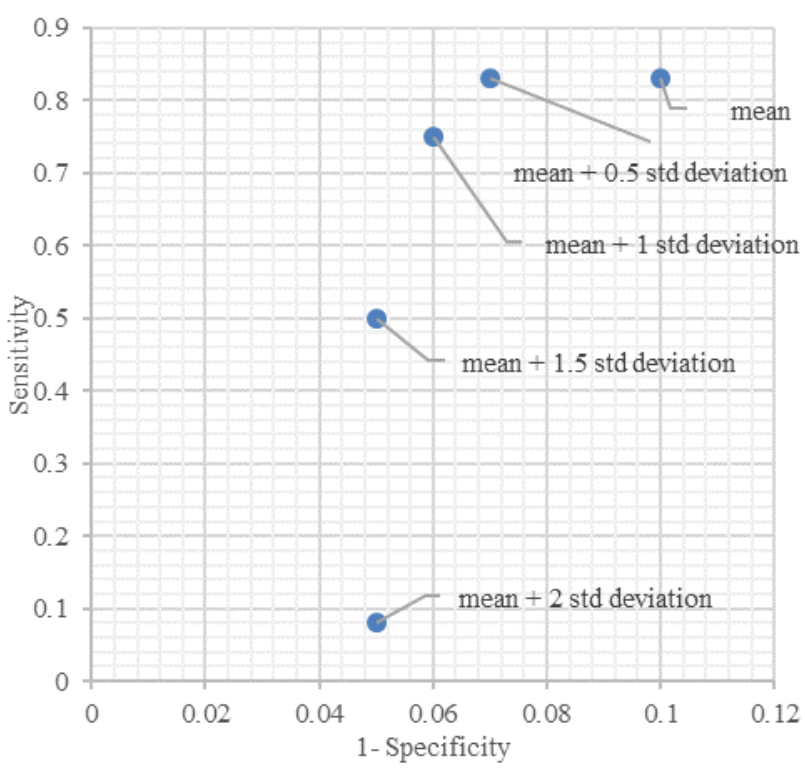

Fig 4. Receiver operating characteristic space showing impacts of different thresholds.

Table 4. Cross-table of the improved simulated forecast results $(n=$ 717; threshold $=$ daily mean +1 standard deviation)

\begin{tabular}{lll}
\hline \multirow{2}{*}{ Maximum demand forecast } & \multicolumn{2}{l}{ Maximum demand observed } \\
\cline { 2 - 3 } & Yes & No \\
\hline Yes & 10 & 35 \\
No & 2 & 670 \\
\hline Sensitivity & 0.83 & \\
\hline Specificity & 0.95 & \\
\hline Precision & 0.22 & \\
\hline Accuracy & 0.95 \\
\hline F-Score & 0.35 \\
\hline
\end{tabular}

\section{Case Studies and Results}

We apply the proposed method to 3 different manufactories. The method takes monthly average as historical reference and forecasts the daily demand peaks in every working days of that month. We have 2 case studies with 15 minutes sampling rate, and one with 1 hour sampling rate to demonstrate the methodology's ability to do the forecasting in different setup. The methodology is run two times to get a better result between using different thresholds, which we specify in the previous section. To show an example we display a monthly mean curve and a daily demand curve in a same figure for the following case studies.

\subsection{Electric/Diesel Generator Enclosures Manufactory (Cast Study 4.1)}

The company employs about 200 people and occupies a $107,300 \mathrm{ft}^{2}$ facility. The plant operates about 6,240 hours annually and produces sheet metal enclosures for generators and large motors and provides testing to those generators and motors. Fig. 5 depicts a March average monthly curve and a daily curve of the March $3^{\text {rd. }}$. Fig. 6 shows a normalized curve after filtering out anything that is below mean plus one standard deviation and its respective ROC. Table 5 shows the forecasting results of 4.1 .
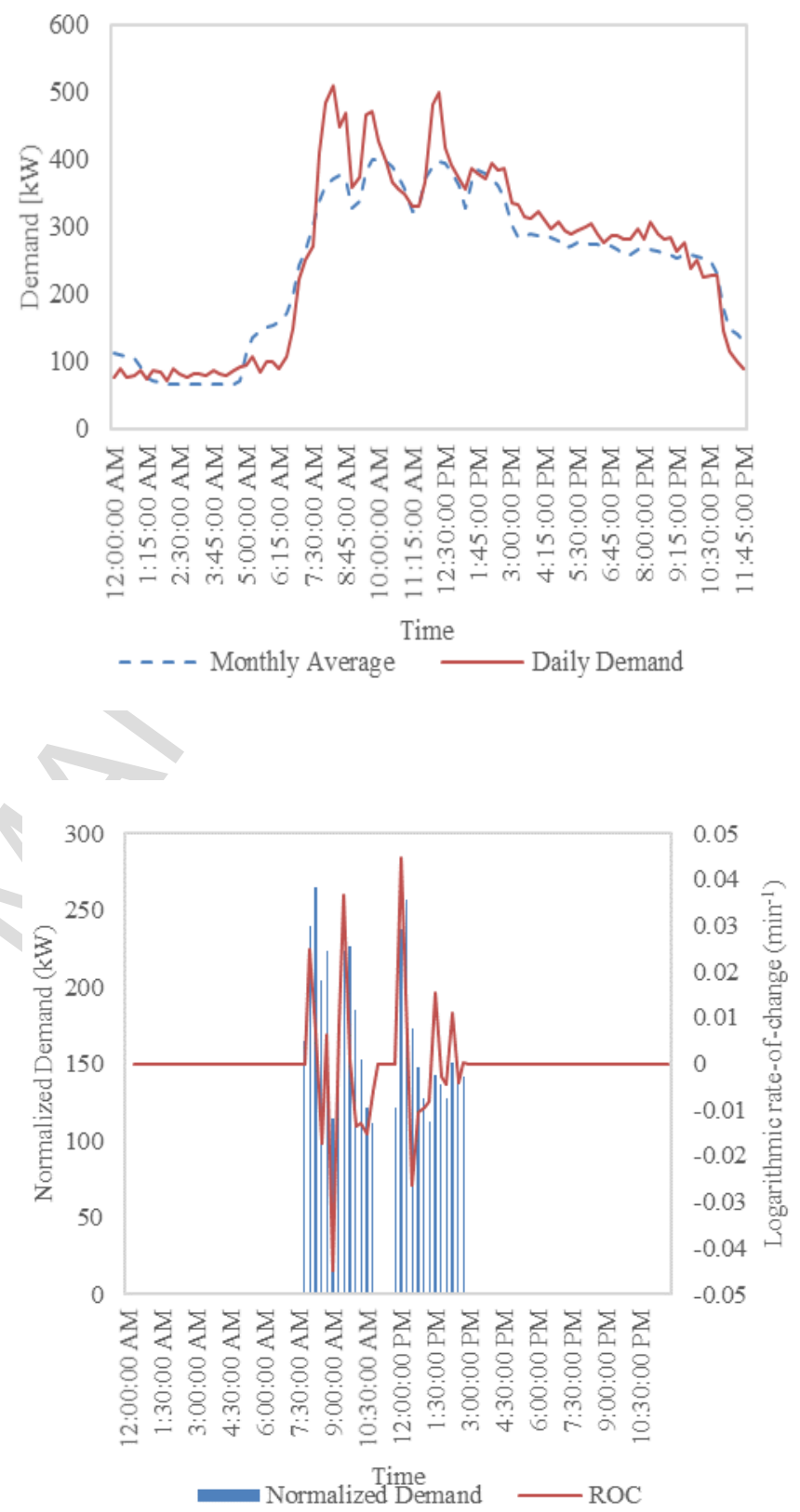

Fig 5. November's monthly average and a daily demand (4.1)

Fig 6. Normalized demand and ROC (4.1) 
Table 5. Cross-table of the forecasting results of case study $4.1(\mathrm{n}=$ 8,$159 ;$ threshold $=$ mean +1 standard deviation; sampling rate $=15$ minutes)

\begin{tabular}{lll}
\hline \multirow{2}{*}{ Maximum demand forecast } & \multicolumn{2}{l}{ Maximum demand observed } \\
\cline { 2 - 3 } & Yes & No \\
\hline Yes & 141 & 390 \\
No & 23 & 7,606 \\
\hline Sensitivity & 0.86 \\
\hline Specificity & 0.95 & \\
\hline Precision & 0.27 & \\
\hline Accuracy & 0.95 & \\
\hline F-Score & 0.41 & \\
\hline
\end{tabular}

\subsection{Metal Part Manufactory (Cast Study 4.2)}

The second case study is for a precision metal parts manufactory, located in Indianapolis. The company operates 7,200 hours annually. The annual electricity consumption is 1.7 million $\mathrm{kWh}$ and average monthly peak demand is $309 \mathrm{~kW}$ as shown in Fig 7. Fig. 8 shows a normalized curve after filtering out anything that is below mean plus one standard deviation and its respective ROC and Table 6 has the results.

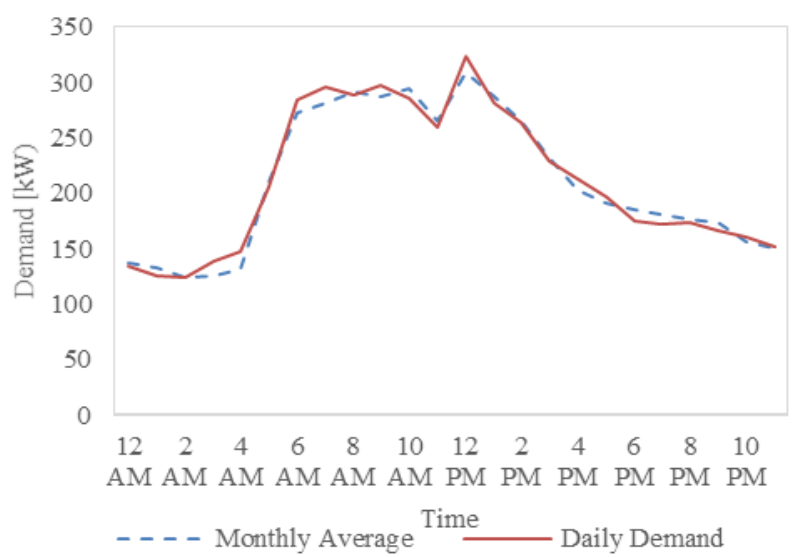

Fig 7. April's monthly average and a daily demand (4.2)

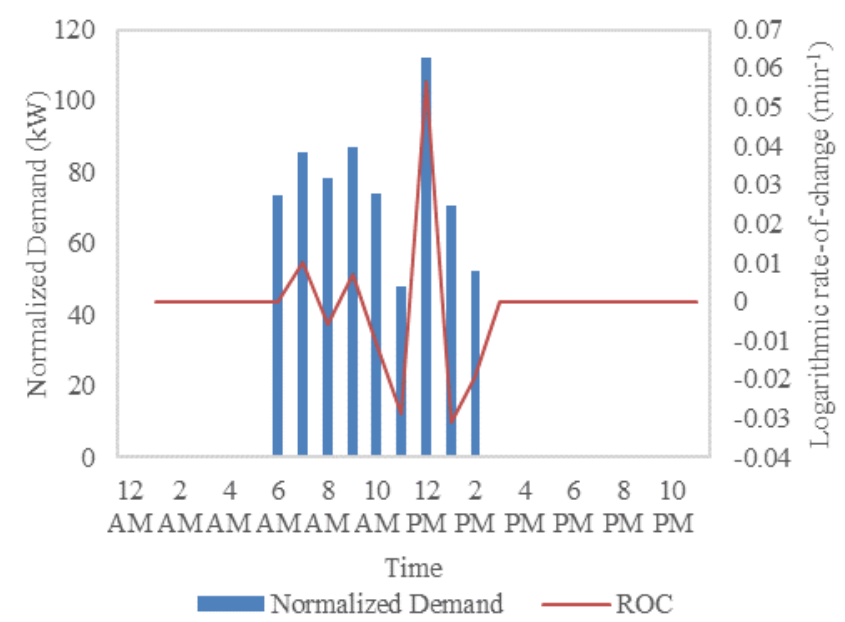

Fig 8. Normalized demand and ROC (4.2)
Table 6. Cross-table of the forecasting results of case study $4.2(\mathrm{n}=$ 3,337 ; threshold $=$ mean +0.5 standard deviation; sampling rate $=1$ hour)

\begin{tabular}{lll}
\hline \multirow{2}{*}{ Maximum demand forecast } & \multicolumn{2}{l}{ Maximum demand observed } \\
\cline { 2 - 3 } & Yes & No \\
\hline Yes & 251 & 128 \\
No & 17 & 2,941 \\
\hline Sensitivity & 0.94 & \\
\hline Specificity & 0.96 & \\
\hline Precision & 0.66 & \\
\hline Accuracy & 0.96 & \\
\hline F-Score & 0.78 & \\
\hline
\end{tabular}

4.3 Aluminum Anodizing Manufactory (Cast Study 4.3)

In 4.3 , the company operates 6,000 hours annually. Its annual electricity usage is 1.04 million $\mathrm{kWh}$ and average monthly peak demand is $197 \mathrm{~kW}$ as shown in Fig. 9. Furthermore, Fig. 10 shows a normalized curve after filtering out anything that is below mean plus one standard deviation and its respective ROC and Table 7 has the results.

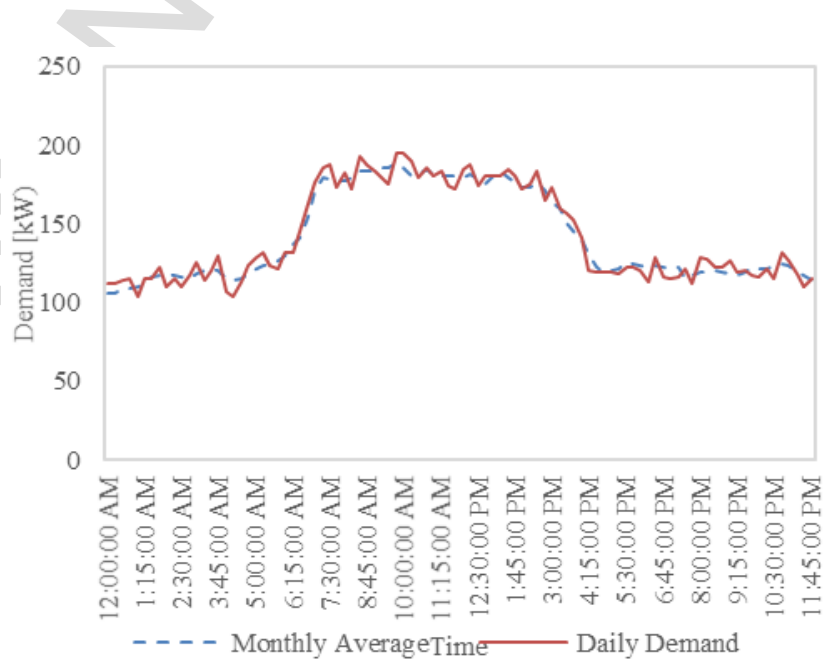

Fig 9. May's monthly average and a daily demand (4.3)

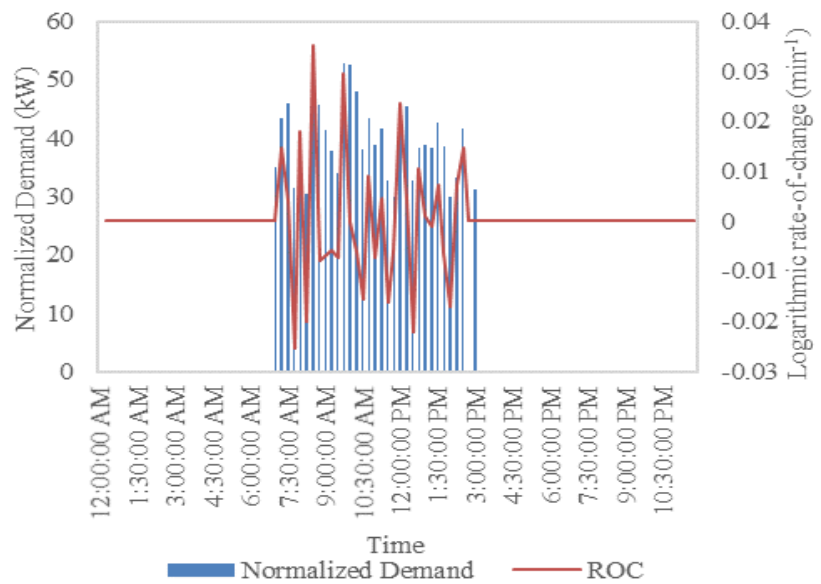

Fig 10. Normalized demand and ROC (4.3) 
Table 7. Cross-table of the forecasting results of case study 4.3 ( $\mathrm{n}=$ 7,030; threshold $=$ mean +1 standard deviation; sampling rate $=15$ minutes)

\begin{tabular}{lll}
\hline \multirow{2}{*}{ Maximum demand forecast } & \multicolumn{2}{l}{ Maximum demand observed } \\
\cline { 2 - 3 } & Yes & No \\
\hline Yes & 213 & 1,012 \\
No & 62 & 5,743 \\
\hline Sensitivity & 0.77 & \\
\hline Specificity & 0.85 & \\
\hline Precision & 0.17 & \\
\hline Accuracy & 0.85 & \\
\hline F-Score & 0.28 & \\
\hline
\end{tabular}

\section{Discussion}

The method is developed using stochastic analysis on the demand and it determines the time of peak by comparing the logarithmic rate-of-change between the data and the historical references. We tested the methodology to three different types of demand patterns. Case Study 4.1 (4.1) had distinct but not unique daily demand peaks. The sampling rate of 4.1 is 15 minutes. Case Study 4.2 (4.2) has a distinct and unique daily peak and the sampling rate is 1 hour. Case Study 4.3 (4.3) has neither distinct nor unique peaks and the sampling rate is 15 minutes. From our numerical simulation example and case studies we have found some strengths and weaknesses of the methodology.

The data quality is an important factor. Case study 4.2 had the highest Fscore meaning the model performs better on this case. Qualitatively, 4.2 has a distinct and unique daily peak and therefore decreased the chance for having false positives (higher specificity). One can also argue that smaller deviation between the daily curve and the monthly average contributes to better result. Moreover, a lower sampling rate can effectively filter noises out, making the peak value more stand-out.

On the other hand, case study 4.1 had distinct but not unique daily demand peaks. The method captured $86 \%$ of the daily maximum but also gave almost 3 times as many false positives as true positives. The reason is that the methodology cannot well distinguish among spikes. Every spike registers a high upward ROC momentum and it will eventually be counted as a positive signal if the upward movement is strong. Further improvement is needed.

Finally, the method performed not as good in 4.3 because the demand pattern constituted neither distinct nor unique peaks. The curve has more randomness within a relatively small range. A slow and plateau-forming average also give a low value of reference ROC, which causes more false positives. F-score ranges were between $0.28-0.78$ and it was 0.43 using aggregate forecasting for all three cases. Table 8 shows the aggregate forecasting results of all case studies.
Table 8. Cross-table of the aggregate forecasting results of all case studies $(n=18,527)$

\begin{tabular}{lll}
\hline \multirow{2}{*}{ Maximum demand forecast } & \multicolumn{2}{l}{ Maximum demand observed } \\
\cline { 2 - 3 } & Yes & No \\
\hline Yes & 605 & 1,530 \\
No & 102 & 16,290 \\
\hline Sensitivity & 0.86 & \\
\hline Specificity & 0.91 & \\
\hline Precision & 0.28 & \\
\hline Accuracy & 0.91 & \\
\hline F-Score & 0.43 & \\
\hline & &
\end{tabular}

\section{Conclusion}

In this manuscript, a method has been proposed for finding the time that the daily peak-load happens. The method relies on the mean-reverting theory of an Ornstein-Uhlenbeck process that an electrical demand possesses. The solution presented in this paper is meant to be used in short-term to medium-term forecasting. This is important in the daily planning of the system operation by giving warning signal to the plant manager to avoid peak demand charges. Nevertheless, the methodology can be improved to be more accurate and inclusive to long-term forecasting needs.

\section{REFERENCES}

[1] IEA. (2015). World Energy Outlook 2015. Paris: International Energy Agency.

[2] Boroojeni, K. G., Amini, M. H., Bahrami, S., Iyengar, S. S., Sarwat, A. I., \& Karabasoglu, O. (2017). A novel multi-time-scale modeling for electric power demand forecasting: From short-term to medium-term horizon. Electric Power Systems Research, 142, 58-73.

[3] Charytoniuk, W., \& Chen, M. S. (2000). Very short-term load forecasting using artificial neural networks. IEEE transactions on Power Systems, 15(1), 263-268.

[4] Laouafi, A., Mordjaoui, M., \& Dib, D. (2014, December). Very shortterm electricity demand forecasting using adaptive exponential smoothing methods. In Sciences and Techniques of Automatic Control and Computer Engineering (STA), 2014 15th International Conference on (pp. 553-557). IEEE.

[5] Laouafi, A., Mordjaoui, M., \& Dib, D. (2015). One-hour ahead electric load forecasting using neuro-fuzzy system in a parallel approach. In Computational Intelligence Applications in Modeling and Control (pp. 95-121). Springer International Publishing.

[6] Gonzalez-Romera, E., Jaramillo-Moran, M. A., \& Carmona-Fernandez, D. (2006). Monthly electric energy demand forecasting based on trend extraction. IEEE Transactions on power systems, 21(4), 1946-1953.

[7] Jaramillo-Morán, M. A., González-Romera, E., \& Carmona-Fernández, D. (2013). Monthly electric demand forecasting with neural filters. International Journal of Electrical Power \& Energy Systems, 49, 253263.

[8] Lee, W. J., \& Hong, J. (2015). A hybrid dynamic and fuzzy time series model for mid-term power load forecasting. International Journal of Electrical Power \& Energy Systems, 64, 1057-1062.

[9] Laouafi, A., Mordjaoui, M., \& Laouafi, F. (2015, May). An evaluation of conventional and computational intelligence methods for medium and long-term load forecasting in Algeria. In Control, Engineering \& Information Technology (CEIT), 2015 3rd International Conference on (pp. 1-6). IEEE. 
[10] Zhang, Z., \& Ye, S. (2011, November). Long term load forecasting and recommendations for china based on support vector regression. In Information Management, Innovation Management and Industrial Engineering (ICIII), 2011 International Conference on (Vol. 3, pp. 597602). IEEE.

[11] Kandil, M. S., El-Debeiky, S. M., \& Hasanien, N. E. (2002). Long-term load forecasting for fast developing utility using a knowledge-based expert system. IEEE transactions on Power Systems, 17(2), 491-496.

[12] Edward, V. (2014). Mean-Reverting Stochastic Models for the Electricity Spot Market.

[13] Stein, E. M., \& Stein, J. C. (1991). Stock price distributions with stochastic volatility: an analytic approach. The review of financial studies, 4(4), 727-752.

[14] Widén, J., \& Wäckelgård, E. (2010). A high-resolution stochastic model of domestic activity patterns and electricity demand. Applied Energy, 87(6), 1880-1892.

[15] Islam, B. U. (2011). Comparison of conventional and modern load forecasting techniques based on artificial intelligence and expert systems. IJCSI International Journal of Computer ScienceIssues, 8(5).

[16] Li, H. Z., Guo, S., Li, C. J., \& Sun, J. Q. (2013). A hybrid annual power load forecasting model based on generalized regression neural network with fruit fly optimization algorithm. Knowledge-Based Systems, 37, 378-387.

[17] Hyndman, R. J., \& Athanasopoulos, G. (2014). Forecasting: principles and practice. OTexts.; pp. 120-125, 2014 Sep 20.

[18] Alkhathami, M. (2015). Introduction to electric load forecasting methods. Journal of Advanced Electrical and Computer Engineering, 2(1), 1-12.

[19] Suganthi, L., \& Samuel, A. A. (2012). Energy models for demand forecasting-A review. Renewable and sustainable energy reviews, 16(2), 1223-1240.

[20] Feinberg, E. A., \& Genethliou, D. (2005). Load forecasting. In Applied mathematics for restructured electric power systems (pp. 269-285). Springer, Boston, MA.

[21] Chikobvu, D., \& Sigauke, C. (2012). Regression-SARIMA modelling of daily peak electricity demand in South Africa. Journal of Energy in Southern Africa, 23(3), 23-30.

[22] Amjady, N. (2001). Short-term hourly load forecasting using time-series modeling with peak load estimation capability. IEEE Transactions on Power Systems, 16(4), 798-805.

[23] Sigauke, C., \& Chikobvu, D. (2010). Daily peak electricity load forecasting in South Africa using a multivariate non-parametric regression approach. ORiON, 26(2).

[24] Fan, C., Xiao, F., \& Wang, S. (2014). Development of prediction models for next-day building energy consumption and peak power demand using data mining techniques. Applied Energy, 127, 1-10.

[25] Kalogirou, S. A. (2000). Applications of artificial neural-networks for energy systems. In Energy Systems (pp. 17-35).

[26] Hippert, H. S., Pedreira, C. E., \& Souza, R. C. (2001). Neural networks for short-term load forecasting: A review and evaluation. IEEE Transactions on power systems, 16(1), 44-55.

[27] Li, P., Li, Y., Xiong, Q., Chai, Y., \& Zhang, Y. (2014). Application of a hybrid quantized Elman neural network in short-term load forecasting. International Journal of Electrical Power \& Energy Systems, 55, 749759.

[28] Abdel-Aal, R. E. (2006). Modeling and forecasting electric daily peak loads using abductive networks. International Journal of Electrical Power \& Energy Systems, 28(2), 133-141.

[29] Amin-Naseri, M. R., \& Soroush, A. R. (2008). Combined use of unsupervised and supervised learning for daily peak load forecasting. Energy conversion and management, 49(6), 1302-1308.

[30] Sun, Z., Li, L., \& Dababneh, F. (2016). Plant-level electricity demand response for combined manufacturing system and heating, venting, and air-conditioning (HVAC) system. Journal of Cleaner Production, 135, $1650-1657$

[31] Almeshaiei, E., \& Soltan, H. (2011). A methodology for electric power load forecasting. Alexandria Engineering Journal, 50(2), 137-144.

[32] Weron, R. (2006). Modeling and Forecasting Electricity Loads. Modeling and Forecasting Electricity Loads and Prices: A Statistical Approach, 67-100.
[33] Tang, C. Y., \& Chen, S. X. (2009). Parameter estimation and bias correction for diffusion processes. Journal of Econometrics, 149(1), 658.

[34] Balvers, R., Wu, Y., \& Gilliland, E. (2000). Mean reversion across national stock markets and parametric contrarian investment strategies. The Journal of Finance, 55(2), 745-772.

[35] Fawcett, T. (2006). An introduction to ROC analysis. Pattern recognition letters, 27(8), 861-874. 
- Novel method to forecast the occurrence of daily maximum demand

- Electrical demand pattern can be regarded as a mean reversion process

- The algorithm requires only the historical demand data

- The algorithm works best on patterns with distinct peak occurrences 